\title{
Reasoning About LLVM Code Using Codewalker
}

\author{
David S. Hardin \\ Advanced Technology Center \\ Rockwell Collins \\ Cedar Rapids, IA, USA \\ david.hardin@rockwellcollins.com
}

\begin{abstract}
This paper reports on initial experiments using J Moore's Codewalker to reason about programs compiled to the Low-Level Virtual Machine (LLVM) intermediate form. Previously, we reported on a translator from LLVM to the applicative subset of Common Lisp accepted by the ACL2 theorem prover, producing executable ACL2 formal models, and allowing us to both prove theorems about the translated models as well as validate those models by testing. That translator provided many of the benefits of a pure decompilation into logic approach, but had the disadvantage of not being verified. The availability of Codewalker as of ACL2 7.0 has provided an opportunity to revisit this idea, and employ a more trustworthy decompilation into logic tool. Thus, we have employed the Codewalker method to create an interpreter for a subset of the LLVM instruction set, and have used Codewalker to analyze some simple array-based C programs compiled to LLVM form. We discuss advantages and limitations of the Codewalker-based method compared to the previous method, and provide some challenge problems for future Codewalker development.
\end{abstract}

\section{Introduction}

In previous work [9] [11], we built a translator from Low-Level Virtual Machine (LLVM) intermediate form [16] to the applicative subset of Common Lisp [15] accepted by the ACL2 theorem prover [12], and performed verification on the translated form using ACL2's automated reasoning capabilities.

LLVM is the intermediate form for many common compilers, including the clang compiler used by Apple OS X and iOS developers. LLVM supports a number of language frontends, and LLVM code generation targets exist for a wide variety of machines, including both CPUs and GPUs. LLVM is a register-based intermediate language in Static Single Assignment (SSA) form [4]. As such, LLVM supports any number of registers, each of which is only assigned once, statically (dynamically, of course, a given register can be assigned any number of times). Andrew Appel has observed that "SSA form is a kind of functional programming" [1]; this observation, in turn, inspired us to build a translator from LLVM to the applicative subset of Common Lisp accepted by the ACL2 theorem prover. Our translator, written in OCaml [5], produced an executable ACL2 specification that was able to support proof-based verification, as well as validation via testing.

The above approach was satisfactory for the technology that we had at hand for use with ACL2 in 2013, but had the obvious weakness of relying on a fair amount of unverified code. The situation changed in late 2014, when J Moore released the initial version of Codewalker, an instruction-set-neutral decompilation-into-logic system, with ACL2 7.0 [18]. Thus, an experiment began in early 2015 to determine whether Codewalker could be used to produce a similar proof environment for LLVM code.

M. Kaufmann and D. Rager (Eds.): ACL2 Workshop 2015 (ACL2 2015).

EPTCS 192, 2015, pp. 79-92 doi 10.4204/EPTCS.192.7 


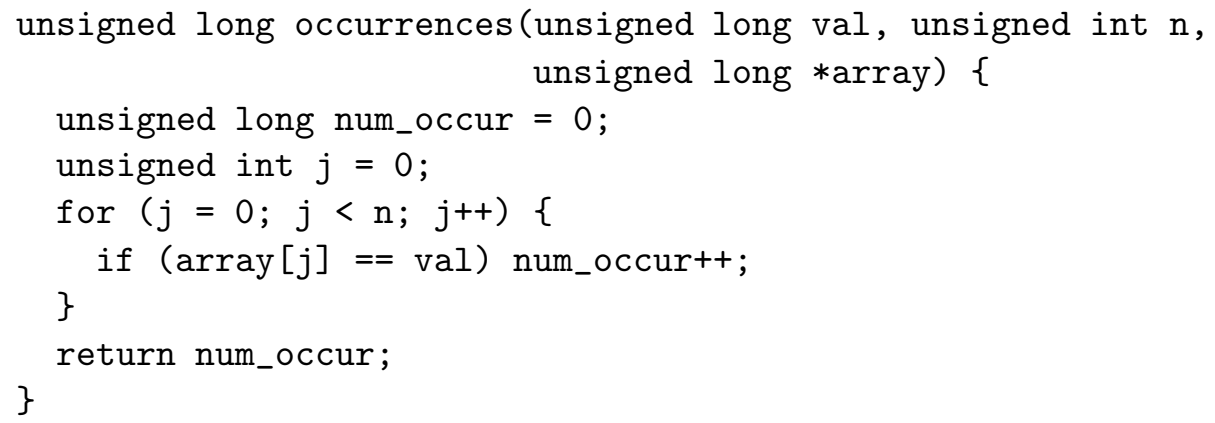

Figure 1: Example $\mathrm{C}$ code to count occurrences of an input value in an array.

\section{An Example}

As an example, consider the $\mathrm{C}$ source code of Figure 1 This function counts the number of occurrences of a given value in the first $\mathrm{n}$ elements of an array. (NB: By default the clang compiler treats all int values as 32 bits wide, and all long values as 64 bits wide.)

This is an admittedly simple example, but it allows us to narrate a complete analysis within the confines of this paper, and should be within Codewalker's capabilities to analyze. We have also performed similar analyses for other small C programs, namely tail-recursive factorial, as well as a program to compute the sum of array elements.

LLVM code for this function is produced by invoking clang as follows: clang $-01-\mathrm{S}$ -emit-llvm occurrences.c. The generated LLVM code for clang version 6.1.0 (which supports LLVM 3.6.0) is excerpted in Figure 2, this is essentially the same code as reported in [9].

Observe that LLVM output is similar to assembly code, with labels and low-level opcodes like br (branch), icmp (integer compare) and load (load from memory). Registers are prepended with the "\%" character, and are given sometimes-meaningful names. Consistent with the SSA philosophy, no register appears on the left hand side of an assignment ("=") more than once. A peculiar feature of LLVM code is the phi instruction, which provides register renaming at a branch target.

\subsection{Translation to ACL2 Syntax}

In previous work, we automatically translated the above LLVM program into an ACL2 functional program. In the current work, we merely translate the LLVM assembly code syntax into a form that is easier for ACL2 to process. The translated form for the LLVM code of Figure 2 is depicted in Figure 3 .

The instruction format is straightforward: if the LLVM instruction is $a=$ ins $b$ c, then the ACL2 syntax is (INS A B C). Thus, (ADD $x$ y $z$ ) stores the sum of the contents of registers (locals) $y$ and $z$ in register $\mathrm{x}$; and ( $\mathrm{BR}$ E F G) branches to the instruction word at the current program counter + offset $F$ if register $\mathrm{E}$ is nonzero, and to the instruction word at the current program counter + offset $\mathrm{G}$ otherwise. A few new instructions have been added to aid in phi processing: (CONST X) pushes a constant value $\mathrm{X}$ on a LIFO stack; (PUSH Y) pushes the contents of register Y onto the stack; and (POPTO Z) pops the top of stack value into register Z. We also define a (HALT) instruction so we don't have to worry about defining a return linkage (this is future work).

Each instruction occupies one instruction word (of indeterminate size), and each register holds an 


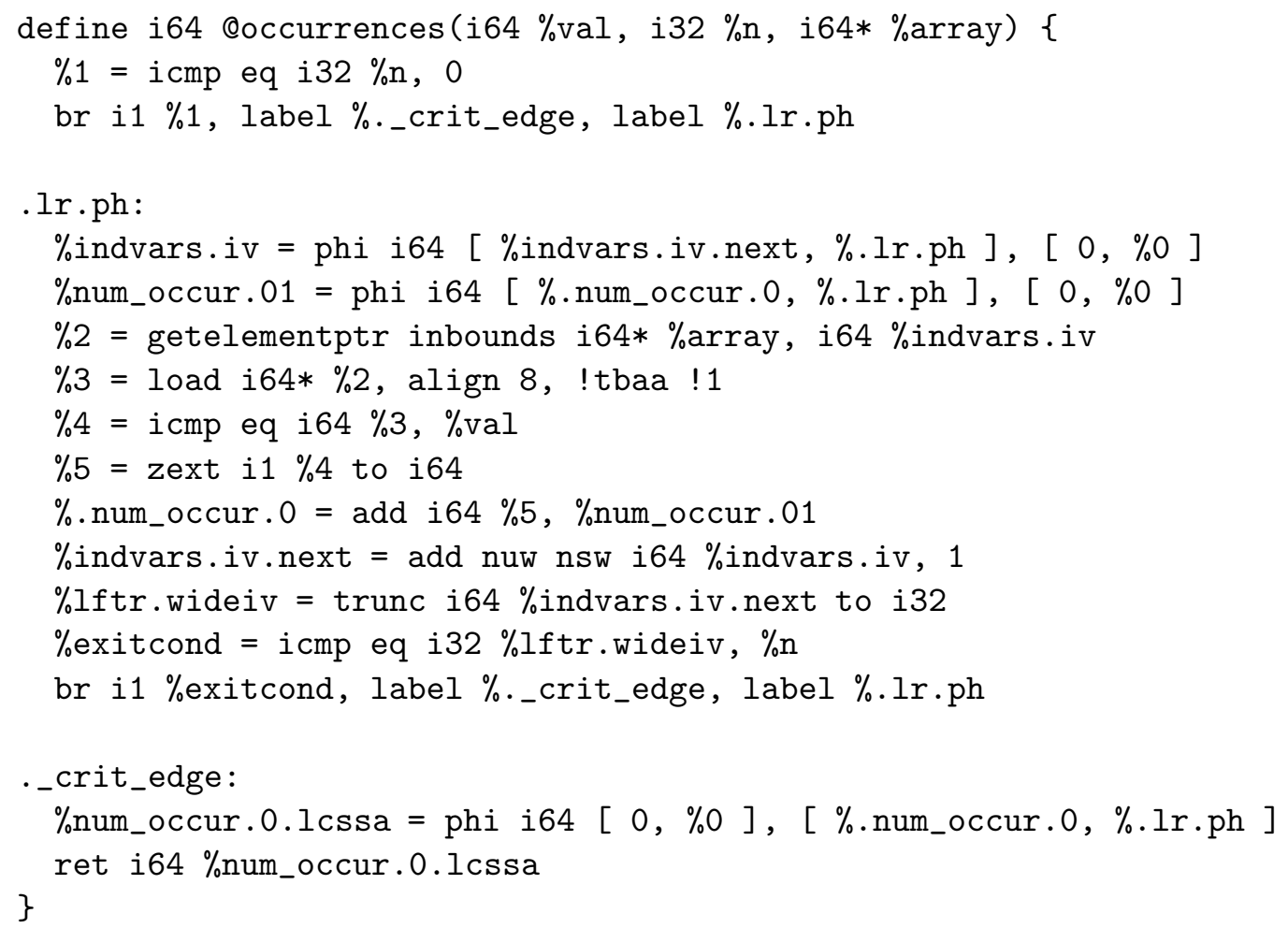

Figure 2: LLVM code for the occurrences example. 


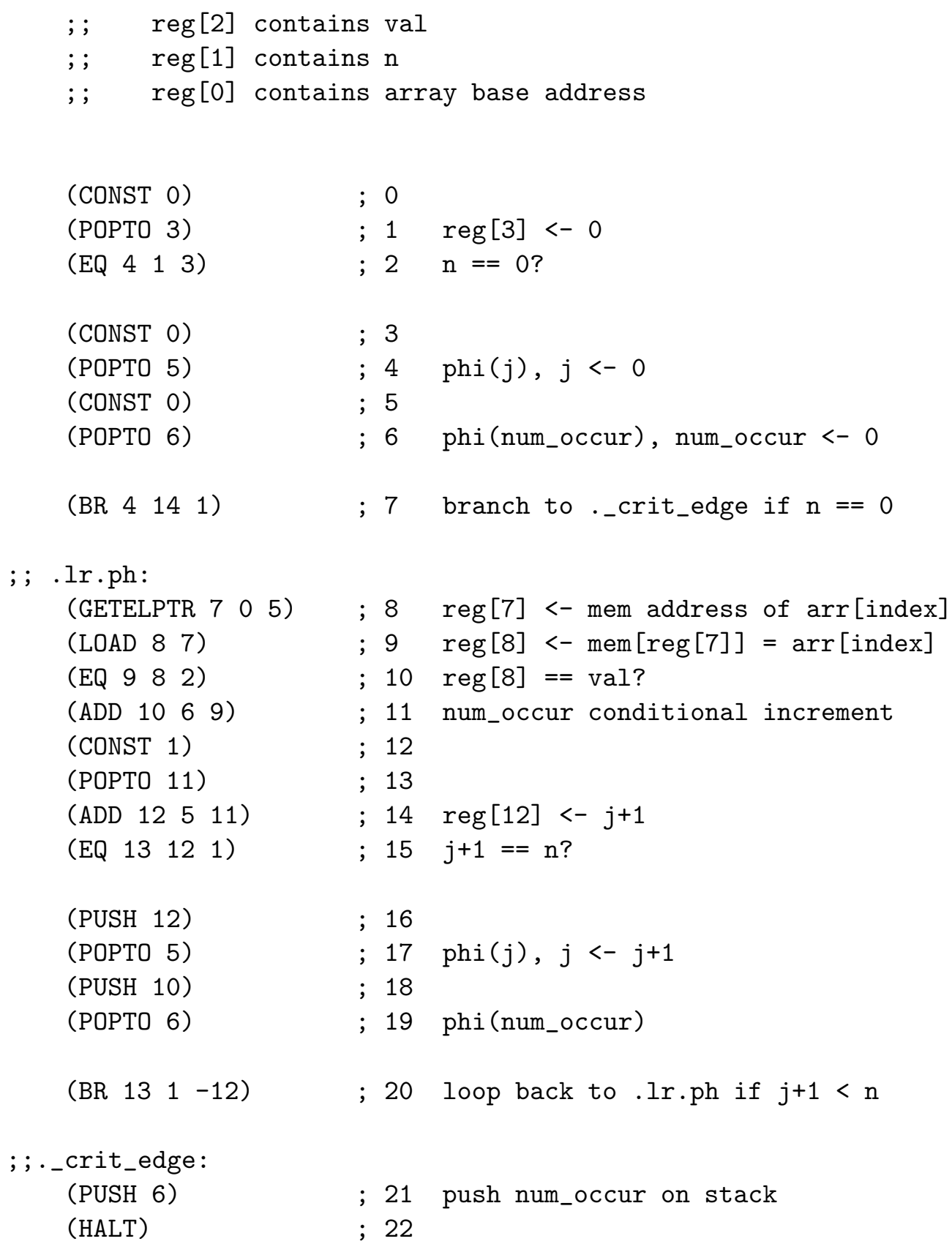

Figure 3: ACL2 representation of the LLVM code for the occurrences example. 
unbounded integer. This represents a slight loss of fidelity relative to the previous work, but we thought it unwise to tackle issues related to both Codewalker and modular arithmetic at the same time.

\section{LL2: An LLVM Subset Interpreter}

Before being able to utilize Codewalker, we must first define an operational semantics, or interpreter, for the target instruction set. The Codewalker sources provide one such example interpreter, for the M1 subset of the Java Virtual Machine (JVM) [14]. We used this ACL2 code as the basis for our LLVM subset interpreter, called LL2. As is typical with such an interpreter written in ACL2, a machine state data structure is declared, and passed as a parameter to all functions that read and/or write elements of the state. If a given function updates the state, the modified state must be returned. Obviously, for a large state, functional update of the state can become quite expensive. Thus, an ACL2 singlethreaded object (stobj) [2] is often used to represent state. The destructive update property of stobjs provides good performance when executing functions on concrete state. The LL2 machine stobj, called simply s, contains fields for the Program Counter (PC), local variables, memory, stack, and program storage. All but the first can be thought of as lists. Accessor and updater functions are defined for all fields, with updaters preceded by a '!' character; thus (loi k s) retrieves the kth local variable (or register, in LLVM parlance), while (!loi $j$ val $s$ )) updates the value of the jth register to val. Note that (loi k s) is defined as (nth k ( $r d$ :locals $s)$ ), and (!loi $j$ val $s$ ) is defined as (wr :locals (update-nth $j$ val (rd :locals $s$ )) $s$ ).

Once the machine state data structure is defined, semantic functions need to be written for all supported instructions. For example, the semantic function for ( $E Q \times y z$ ) is as follows:

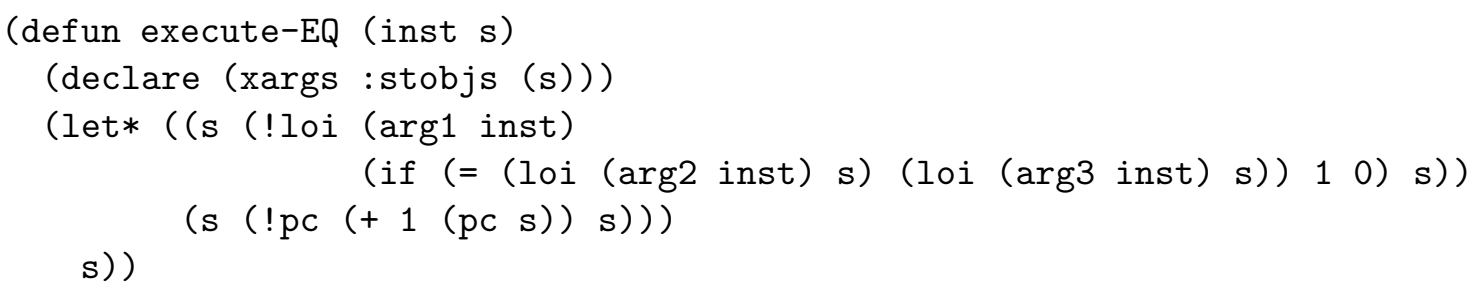

where inst is the list form of an instruction (as depicted in Figure 3), (arg1 inst) is (nth 1 inst), (arg2 inst) is ( $n$th 2 inst), and (arg3 inst) is (nth 3 inst). Thus, execute-EQ stores the value 1 in the register indicated by the first argument if the value stored in the register indicated by the second argument is equal to the value stored in the register indicated by the third argument; the value 0 is stored in the first argument register otherwise. Finally, the program counter is incremented.

Once semantic functions have been written for every supported instruction, a simple instruction selector function can be composed, as follows:

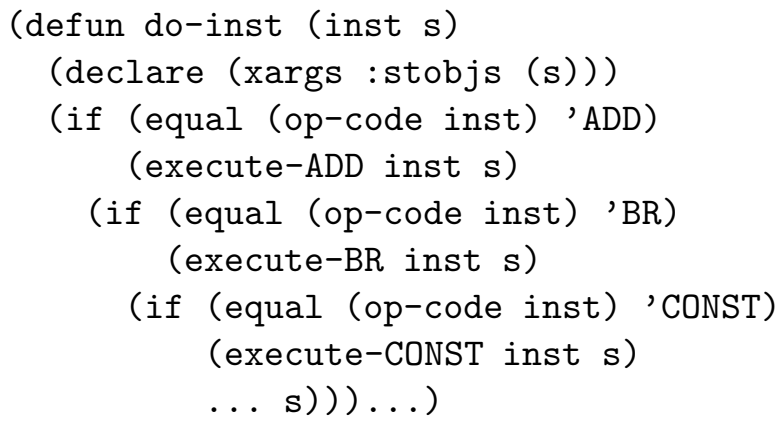


This instruction selector function is called by the instruction stepper function:

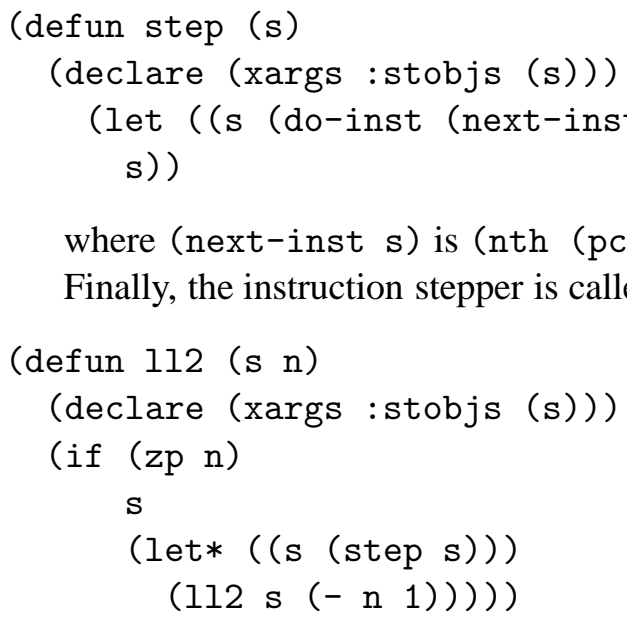

Note that this is all fairly standard technique for defining an instruction set interpreter in ACL2; one peculiarity, however, is that the top-level interpreter argument order (namely, state followed by step count) is mandated by Codewalker.

\subsection{Concrete Execution}

It is advantageous to be able to validate LLVM programs by running them against concrete inputs. Since all of our interpreter functions are executable, we can readily perform such validation testing. In the ACL2 code of Figure 4, we set up an initial state, establishing an array of length 8 starting at address 100. We write various values into memory at increasing addresses. The array base address is stored in local 0 , followed by the $\mathrm{n}$ and val parameters, in locals 1 and 2, respectively. The program is written using the (wr :program ' (...)) form. The program is stepped to conclusion by invoking (112 s 113); the return value can be found at (loi $6 \mathrm{~s}$ ).

As we have written the value 399 into the array three times, when we run the interpreter and fetch the return result as described above, we obtain the expected value: 3 . The interpreter executes approximately 226,000 LLVM instructions per second on an ordinary laptop computer. This is approximately one-tenth the speed of our previous method, as is to be expected for an interpreted vs. compiled approach, but this performance level is still more than adequate for validation testing.

\section{Codewalker}

Now that the interpreter for LL2 is in place, we can begin to use Codewalker to perform decompilation into logic for LLVM programs, producing semantic functions for those programs that the ACL2 user can further reason about. The end goal is to prove that the LLVM code for a given function implements a much more abstract function, written in ACL2, about which we can readily prove interesting correctness properties. In the extensive code documentation for Codewalker, the system is described as follows [18]:

Two main facilities are provided by Codewalker: the abstraction of a piece of code into an ACL2 "semantic function" that returns the same machine state, and the "projection" of such a function into another function that computes the final value of a given state component using only the values of the relevant initial state components. 


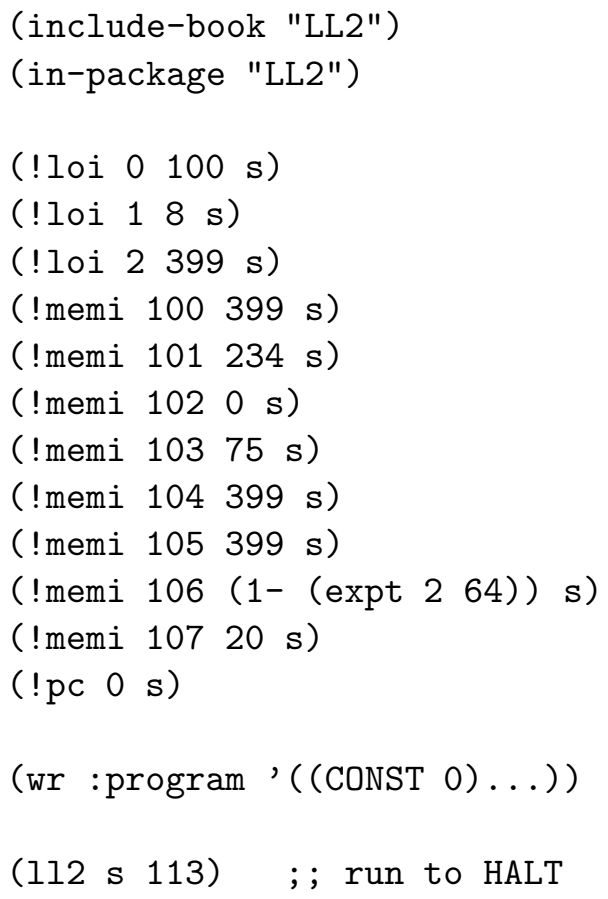

Figure 4: Concrete test case for the occurrences example.

Codewalker is independent of any particular machine model, as long as a step-based operational semantics for the machine is defined in ACL2. To facilitate this language-independent analysis, the user must declare a "model API" that allows Codewalker to access functionality of the model (e.g., setting the pc in a symbolic state). Generally speaking, Codewalker accesses the model by forming symbolic ACL2 expressions that answer certain questions, then applying the ACL2 simplifier with full access to user-proved lemmas, and then inspecting the resulting term to recover the answer.

Thus, to begin, we tell Codewalker about our operational semantics using def-model-api, telling it the name of our interpreter function, the state variable, whether the state is a stobj, the name of the step function, and so on. We next introduce the program to be analyzed, and prove some simple theorems about it, e.g. that writes to state fields other than the program field don't affect the program.

Next, we provide Codewalker with important program-level invariants as well as loop invariants. We also assist the system by providing a measure for the loop clock function, as illustrated in Figure 5 ,

Finally, we set Codewalker to work, by invoking its def-semantics function. First, we ask Codewalker to generate a semantic function for the "preamble" of the code (before the loop), then ask it to produce a semantic function for the loop itself, as shown in Figure 6 We often wish to break up the processing in this way, and not give the entire function to Codewalker in a single chunk. One reason for this is that it can be tricky to craft just the right invariants that are true for preamble, as well as the loop and postlude, and that Codewalker will be able to process successfully.

Codewalker development is still in its early phase, and the system is a bit "touchy" when it comes to the combination of focus regions, invariants, measure annotations, and so on that will result in success. In Codewalker's defense, it is very sophisticated software attempting a very difficult job. To quote the 


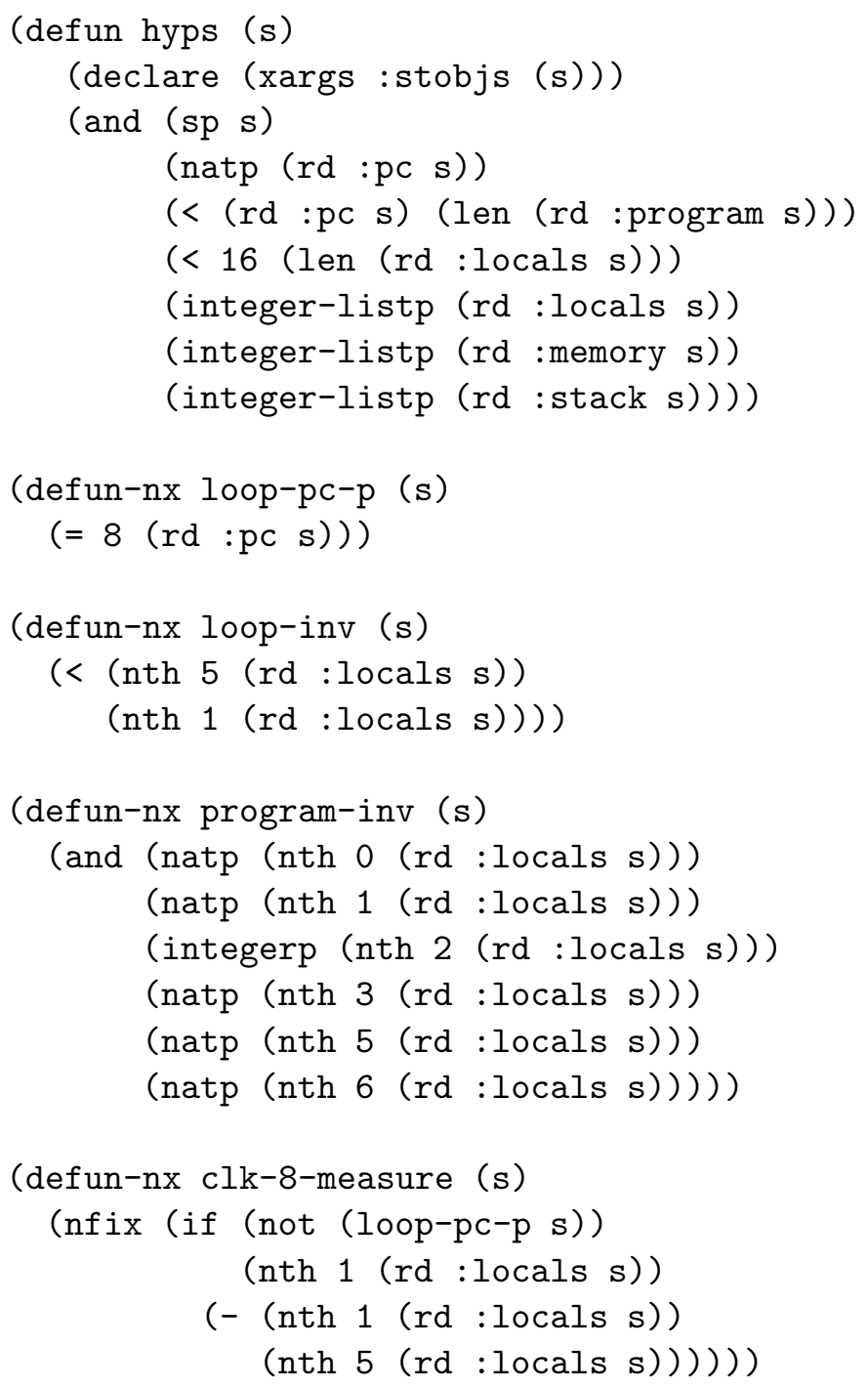

Figure 5: Some invariants and measures provided to Codewalker. 


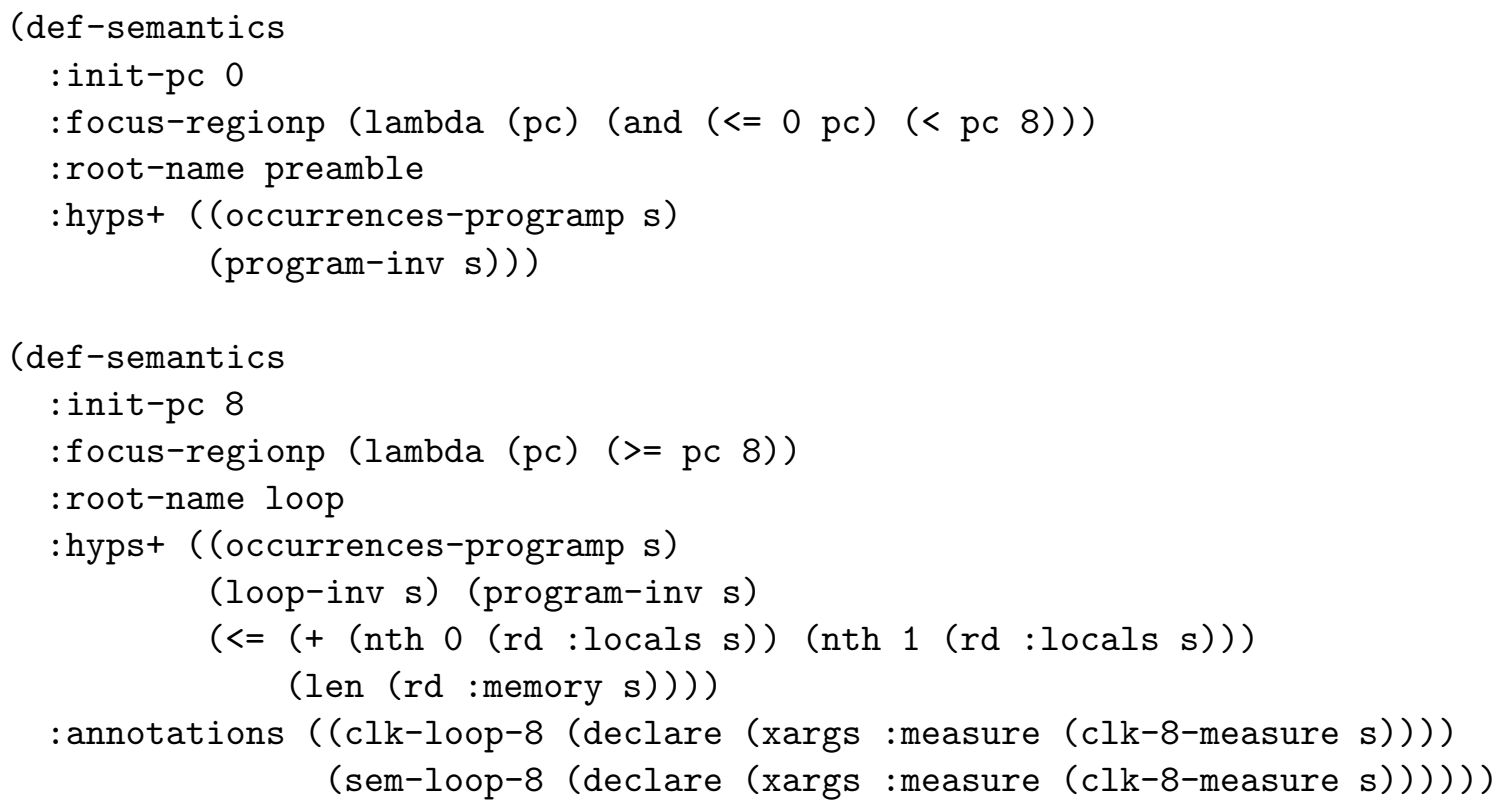

Figure 6: Invocations of Codewalker def-semantics for the occurrences example.

Codewalker documentation: "Def-semantics actually prints a lot of stuff as it goes. It also often fails! Some of its error messages make supposedly helpful suggestions as to what's 'wrong.' Often your response will be to prove more lemmas because things aren't being reduced to the canonical forms. Another response might be to restrict the focus region or strengthen the invariant so as to avoid certain cases." [18]

Codewalker produces decompilations of the indicated code segments, which we can then assemble using functional composition, e.g.:

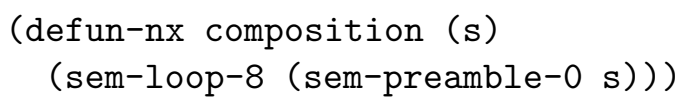

Codewalker also produces correctness theorems about the generated semantics functions, e.g.:

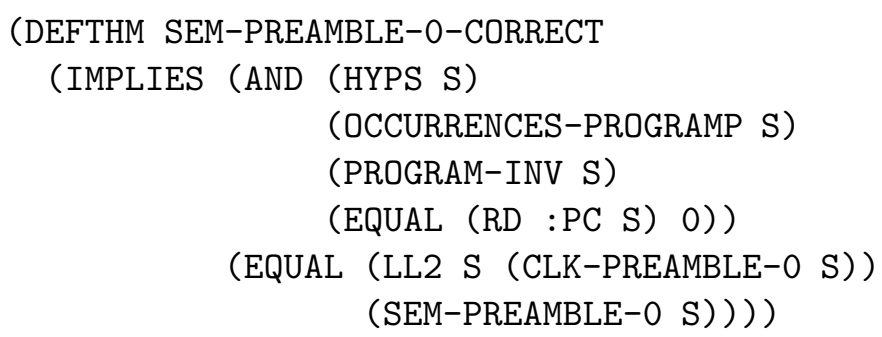




$$
\begin{gathered}
\left(<=\begin{array}{c}
(+ \text { NTH } 0(\text { RD :LOCALS S })) \\
(\text { NTH } 1(\text { RD :LOCALS S }))) \\
(\text { LEN }(\text { RD : MEMORY S })))
\end{array}\right. \\
(\text { EQUAL }(\text { RD :PC S S 8) })
\end{gathered}
$$

(EQUAL (LL2 S (CLK-LOOP-8 S))

$($ SEM-LOOP-8 S ))) )

The latter theorem states that if the LL2 interpreter is poised at the top of the loop $(\mathrm{pc}=8)$ then running the LL2 interpreter with the occurrences program loaded for a proper number of steps (given by (CLK-LOOP-8 S) yields the same result as executing the generated semantic function.

\section{Reasoning about LLVM Code via Codewalker Semantic Functions}

In order to reason about a function such as occurrences in ACL2, we first need to perform abstraction on the data types; particularly, we wish to abstract the input array to a Lisp list. Since we are utilizing stobjs, however, this abstraction has already been provided for us. (Recall that stobjs provide a list abstraction for array data types that feature an efficient, in-place, destructive implementation.)

Next, we need a "golden" list-based specification of occurrences. This function should be easy to reason about using ACL2, and so should be written in non-tail-recursive style, as in the following:

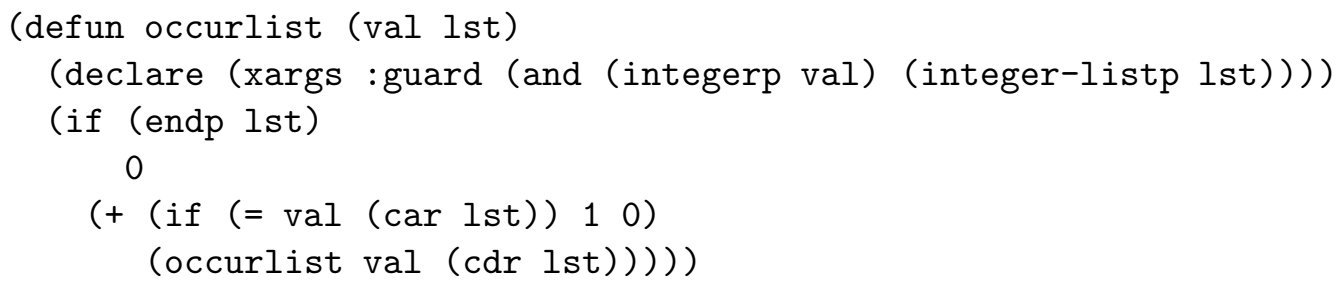

We wish to prove that the execution of the LLVM instructions of the compiled occurrences function operating over an array in memory produces a result equal to the occurlist function operating over a list. Unfortunately for the proof of the above, the semantic functions generated by Codewalker are tail-recursive. The proof actually proceeds by the use of two additional functions, a pair of tailrecursive/non-tail-recursive functions that are generated and proved equal by defiteration, a book found in centaur/misc in the standard ACL2 distribution. (This technique was earlier described in [10].) The call to defiteration is as follows:

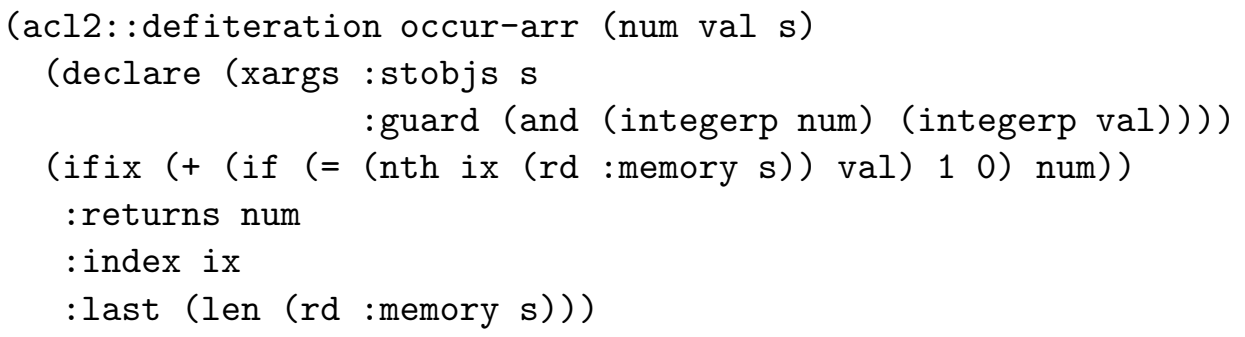

We first prove that the value stored in the num_occur register (register 6) after execution of the composition of semantic functions generated by Codewalker is equal to the result of the tail-recursive function generated by the call to defiteration above: 


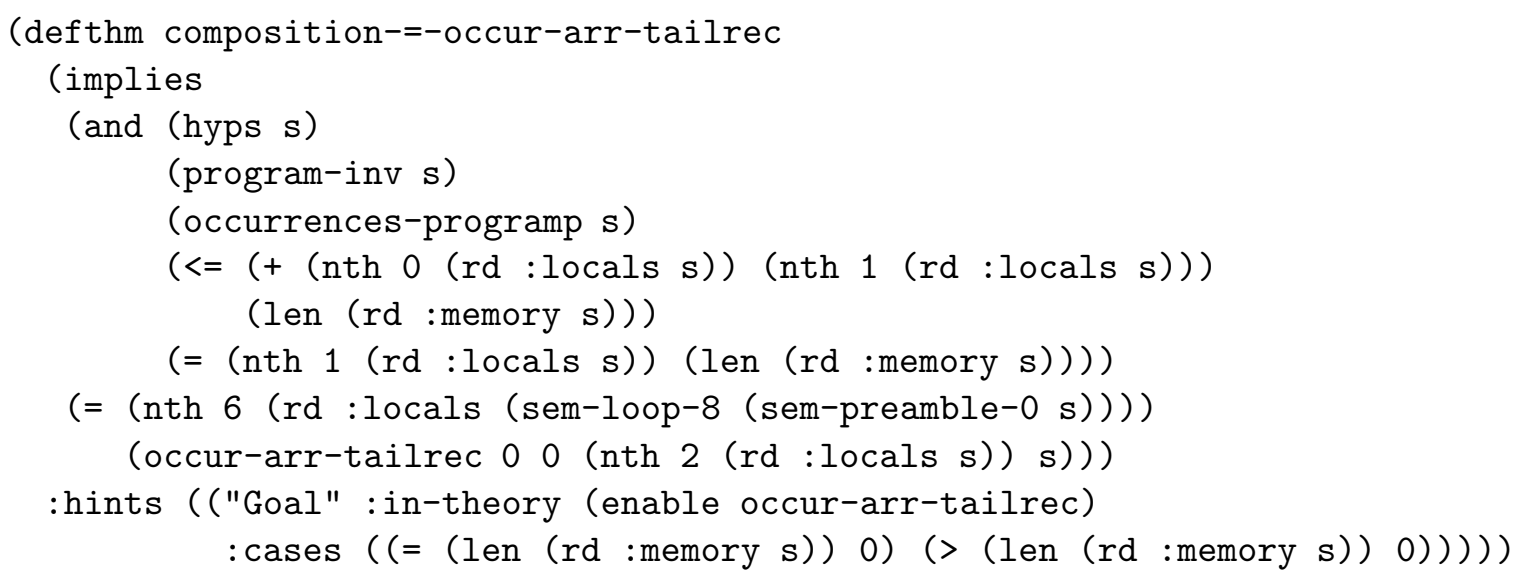

We then prove that the non-tail-recursive function generated by defiteration is equal to occurlist:

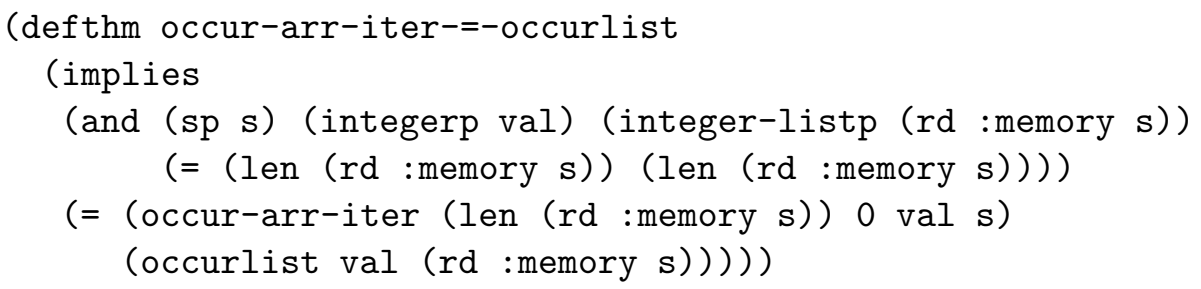

The above theorem can be proved by first proving the following lemma:

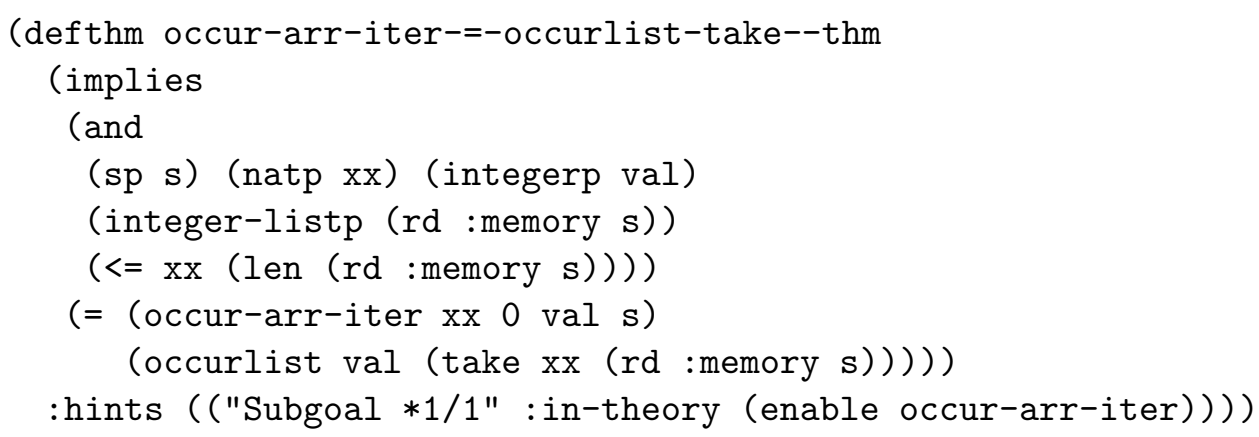

Since occur-arr-iter and occur-arr-tailrec are already proved equal by defiteration, the proof of composition-=-occurlist then follows readily.

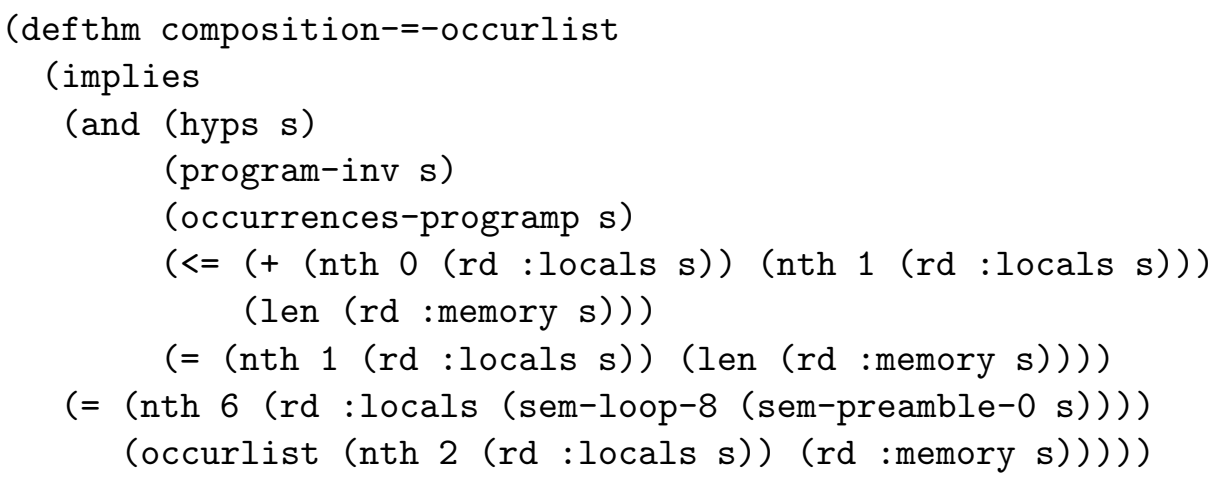

Finally, given the semantic function correctness theorems generated by Codewalker (namely, SEM-PREAMBLE-0-CORRECT and SEM-LOOP-8-CORRECT, the desired final theorem, depicted in Figure 7 can be stated and proved. 


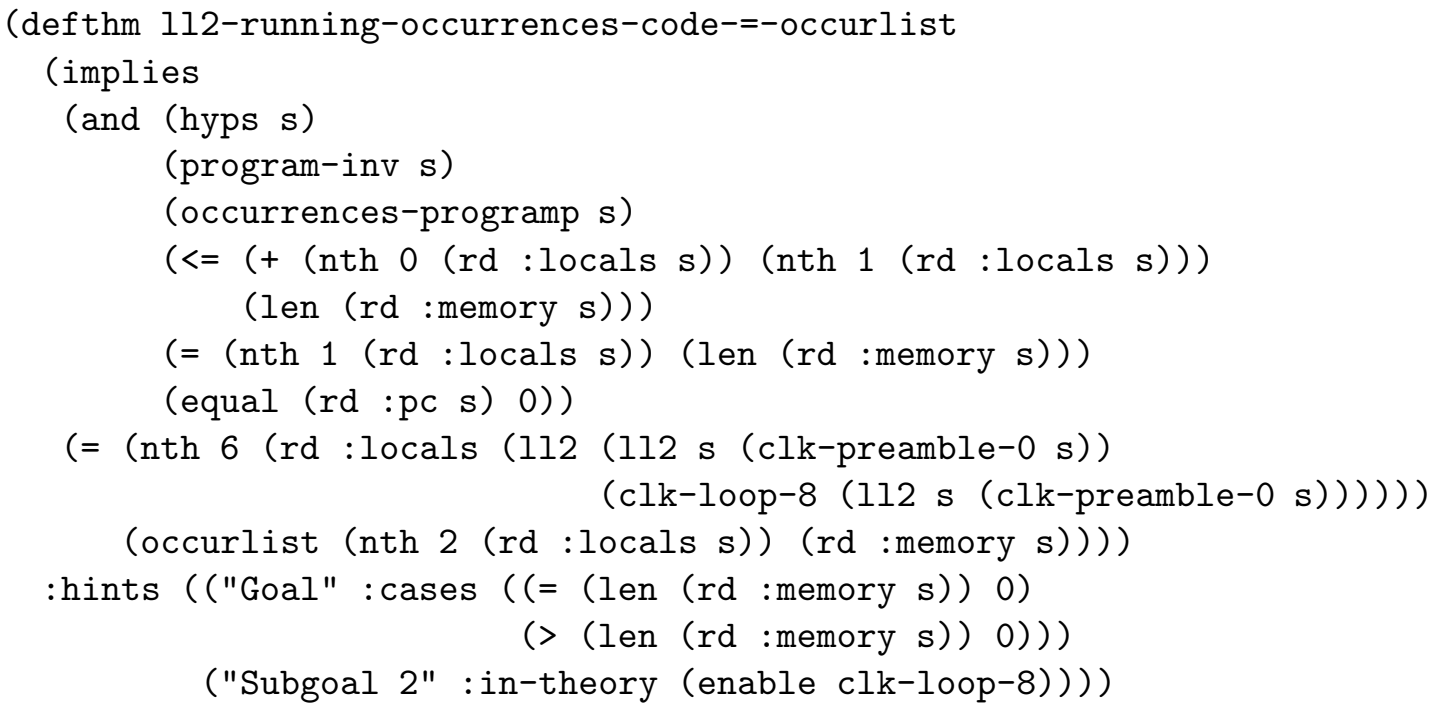

Figure 7: Final theorem, equating the result of executing the LLVM instructions for the occurrences program to its abstract "golden" specification.

\section{Related Work}

The technique of compiling to a Virtual Machine instruction set has made a significant comeback in the past twenty years, starting with the JVM, and continuing with Microsoft's CIL, Android Dalvik, and LLVM. Our work on verification at the virtual machine instruction set level was inspired by J Moore's pioneering work on JVM verification [17], as well as Eric Smith's more recent Axe system, which was used to verify a number of Java cryptographic programs at the bytecode level [20].

Zhao et al. [23] produced several different formalizations of operational semantics for LLVM in Coq [21], noting that their intention is to produce a verified LLVM compiler, similar to the CompCert verified compiler due to Leroy [13] (CompCert does not utilize the LLVM intermediate form). The goal of Zhao et al. was not to produce a verification environment for LLVM bitcode, unlike the present work, but rather to prove the correctness of compiler passes that manipulate LLVM. Jules Villard at Imperial College London is developing llStar, a formal analysis tool for LLVM bitcode. Villard's work so far has focused on proving properties of small LLVM programs that manipulate algebraic data types, utilizing the coreStar symbolic execution engine, separation logic, and SMT technology [22]. LLBMC [7] is a bounded model checker used in bug-finding for $\mathrm{C}$ programs that operates on LLVM bitcode. Similary, KITTeL [6] performs termination analysis on C programs by examining LLVM bitcode. Finally, KLEE [3] is a symbolic execution tool that operates on LLVM bitcode to produce coverage test cases and find bugs in $\mathrm{C}$ programs.

Codewalker was directly influenced by Magnus Myreen's "decompilation into logic" work [19]. It would be interesting to attempt to replicate the work done here using a combination of Myreen's decompiler and Anthony Fox's L3 instruction set description language [8]. 


\section{Conclusion and Future Work}

We have used Codewalker, an instruction-set-neutral decompilation-into-logic system included with the ACL2 theorem prover, to formally analyze C programs that have been compiled to the LLVM intermediate form. Work began by defining a stobj-based interpreter for a subset of the LLVM instruction set, guided by an existing interpreter for the M1 subset of the Java Virtual Machine. Several C programs, including programs to compute factorial, sum of array elements, and number of occurrences of a value in an array, were compiled to LLVM form, and hand-translated to an ACL2-friendly form that could be fed to the interpreter. Validation testing was then conducted on these programs using concrete inputs, before the programs were given to Codewalker for formal analysis. Program-wide invariants, as well as loop invariants and clock measure functions, were defined in order to help Codewalker create semantic functions for program code segments. The composition of these semantic functions was then proved equivalent to more abstract functions: first to a tail-recursive form; then to a non-tail-recursive form (the equality of the latter two having previously been established by the defiteration facility); and finally to a top-level non-tail-recursive "golden" specification. Thus, we were able to prove that several sample LLVM programs implement the top-level specifications for those programs.

Future work will focus on using Codewalker to analyze more complex $\mathrm{C}$ functions, in particular functions that feature nested loops, as well as functions that employ runtime-allocated memory. We have successfully processed the "straight-line" segments for an LLVM insertion sort program (which features a nested loop) using Codewalker, but have not yet successfully composed the generated semantic functions into a whole program for further analysis. Additionally, now that basic programs operating on unbounded integers have been successfully analyzed using Codewalker, a new version of the LLVM interpreter should be developed that can support different finite data word sizes, as well as the LLVM call and ret instructions. Finally, we would like to apply Codewalker to additional instruction set architectures, focusing on physical ISAs, as opposed to virtual ISAs like LLVM.

\section{Acknowledgments}

Many thanks to J Moore for developing Codewalker. I also wish to express my appreciation to J and Matt Kaufmann for several emails that clarified my understanding of Codewalker's capabilities. Thanks also to the anonymous reviewers for their helpful comments. Finally, I wish to acknowledge the wonderful support of my wife, Lori Hardin.

\section{References}

[1] Andrew W. Appel (1998): SSA is Functional Programming. In: SIGPLAN Notices, 33, ACM, pp. 17-20, doi: $10.1145 / 278283.278285$.

[2] Robert S. Boyer \& J Strother Moore (2002): Single-Threaded Objects in ACL2. PADL 2002, doi:10.1007/ 3-540-45587-6_3.

[3] Cristian Cadar, Daniel Dunbar \& Dawson Engler (2008): KLEE: Unassisted and Automatic Generation of High-coverage Tests for Complex Systems Programs. In: Proceedings of the 8th USENIX Conference on Operating Systems Design and Implementation, OSDI'08, USENIX Association, pp. 209-224. Available at http://dl.acm.org/citation.cfm?id=1855741.1855756.

[4] Ron Cytron, Jeanne Ferrante, Barry K. Rosen, Mark N. Wegman \& F. Kenneth Zadeck (1991): Efficiently Computing Static Single Assignment Form and the Control Dependence Graph. In: TOPLAS, 13, ACM, pp. 451-490, doi:10.1145/115372.115320. 
[5] Damien Doligez, Alain Frisch, Jacques Garrigue, Didier Remy \& Jerome Vouillon (2014): The OCaml System Release 4.02 Documentation and Users Guide. http://caml.inria.fr/distrib/ocaml-4.02/ ocaml-4.02-refman.pdf.

[6] Stephan Falke, Deepak Kapur \& Carsten Sinz (2011): Termination Analysis of C Programs Using Compiler Intermediate Languages. In: Proceedings of the 22nd International Conference on Rewriting Techniques and Applications (RTA '11), pp. 41-50, doi:10.4230/LIPIcs.RTA.2011.41.

[7] Stephan Falke, Florian Merz \& Carsten Sinz (2013): The Bounded Model Checker LLBMC (Tool Demonstration). In: Proceedings of the 28th International Conference on Automated Software Engineering (ASE '13).

[8] Anthony Fox (2012): Directions in ISA Specification. In: ITP 2012, doi:10.1007/978-3-642-32347-8_ 23.

[9] David S. Hardin, Jennifer A. Davis, David A. Greve \& Jedidiah R. McClurg (2014): Development of a Translator from LLVM to ACL2. In F. Verbeek \& J. Schmaltz, editors: Proceedings of the 12th International Workshop on the ACL2 Theorem Prover and its Applications, 152, EPTCS, pp. 163 - 177, doi:10.4204/ EPTCS.152.13.

[10] David S. Hardin \& Samuel S. Hardin (2013): ACL2 Meets the GPU: Formalizing a CUDA-based Parallelizable All-Pairs Shortest Path Algorithm in ACL2. In R. Gamboa \& J. Davis, editors: Proceedings of the 11th International Workshop on the ACL2 Theorem Prover and its Applications, 114, EPTCS, pp. 127 - 142, doi:10.4204/EPTCS.114.10.

[11] David S. Hardin, Jedidiah R. McClurg \& Jennifer A. Davis (2013): Creating Formally Verified Components for Layered Assurance with an LLVM-to-ACL2 Translator. In: Proceedings of the 2013 Layered Assurance Workshop, ACM.

[12] Matt Kaufmann, Panagiotis Manolios \& J Strother Moore (2000): Computer-Aided Reasoning: An Approach. Kluwer Academic Publishers, doi:10.1007/978-1-4757-3188-0.

[13] Xavier Leroy (2009): Formal Verification of a Realistic Compiler. In: Communications of the ACM, 52, pp. 107-115, doi:10.1145/1538788.1538814.

[14] Tim Lindholm, Frank Yellin, Gilad Bracha \& Alex Buckley: The Java Virtual Machine Specification, Java SE 8 Edition. https://docs.oracle.com/javase/specs/jvms/se8/jvms8.pdf.

[15] LispWorks Ltd.: Common Lisp HyperSpec. http://www.lispworks .com/documentation/HyperSpec/ Front/index.htm.

[16] LLVM Project: The LLVM Compiler Infrastructure. http://1lvm.org/.

[17] J Strother Moore (1999): Proving Theorems about Java-like Byte Code. In E.-R. Olderog \& B. Steffen, editors: Correct System Design - Recent Insights and Advances, Lecture Notes in Computer Science 1710, Springer-Verlag, pp. 139-162, doi:10.1007/3-540-48092-7_7.

[18] J Strother Moore (2014): Codewalker source code. Standard ACL2 distribution at http://www.cs. utexas.edu/users/moore/acl2.

[19] Magnus O. Myreen, Michael J. C. Gordon \& Konrad L. Slind (2012): Decompilation into Logic - Improved. In: FMCAD'12, ACM/IEEE.

[20] Eric Smith (2011): Axe: An Automated Formal Equivalence Checking Tool for Programs. Ph.D. thesis, Stanford University.

[21] The Coq Development Team (2015): The Coq Proof Assistant Reference Manual, Version 8.4pl6. https:// coq.inria.fr/distrib/current/files/Reference-Manual.pdf.

[22] Jules Villard (2013): Here be wyverns! Verifying LLVM bitcode with llStar. Unpublished manuscript at http://www.doc.ic.ac.uk/ jvillar1/pub/llstar-draft-oct13.pdf.

[23] Jianzhou Zhao, Santosh Nagarakatte, Milo M. K. Martin \& Steve Zdancewic (2012): Formalizing the LLVM Intermediate Representation for Verified Program Transformations. In: POPL'12, ACM, doi:10.1145/ 2103621.2103709. 